Delft University of Technology

\title{
Investigation of 3D Shock Control Bumps for Transonic Buffet Alleviation
}

D'Aguanno, A.; Schrijer, F.F.J.; van Oudheusden, B.W.

DOI

10.2514/6.2021-2558

Publication date

2021

Document Version

Final published version

Published in

AIAA AVIATION 2021 FORUM

\section{Citation (APA)}

D'Aguanno, A., Schrijer, F. F. J., \& van Oudheusden, B. W. (2021). Investigation of 3D Shock Control Bumps for Transonic Buffet Alleviation. In AIAA AVIATION 2021 FORUM [AIAA 2021-2558] (AIAA Aviation and Aeronautics Forum and Exposition, AIAA AVIATION Forum 2021). https://doi.org/10.2514/6.2021-2558

\section{Important note}

To cite this publication, please use the final published version (if applicable).

Please check the document version above.

\section{Copyright}

Other than for strictly personal use, it is not permitted to download, forward or distribute the text or part of it, without the consent of the author(s) and/or copyright holder(s), unless the work is under an open content license such as Creative Commons.

\section{Takedown policy}

Please contact us and provide details if you believe this document breaches copyrights.

We will remove access to the work immediately and investigate your claim. 
Green Open Access added to TU Delft Institutional Repository

'You share, we take care!' - Taverne project

https://www.openaccess.nl/en/you-share-we-take-care

Otherwise as indicated in the copyright section: the publisher is the copyright holder of this work and the author uses the Dutch legislation to make this work public. 


\title{
Investigation of 3D Shock Control Bumps for Transonic Buffet Alleviation
}

\author{
A. D’Aguanno ${ }^{1 *}$, F.F.J. Schrijer ${ }^{1}$ and B.W. Van Oudheusden ${ }^{1}$ \\ ${ }^{\prime}$ : Aerodynamics Sect. (AWEP), Delft University of Technology, 2629 HS Delft, The Netherlands \\ * Correspondent author: A.Daguanno@tudelft.nl
}

\begin{abstract}
This experimental study investigates the use of shock control bumps (SCBs) for controlling transonic buffet. Three-dimensional SCBs have been applied on the suction side of an OAT15A supercritical airfoil with the experiments conducted in the transonic-supersonic wind tunnel of TU Delft for fully developed buffet conditions $\left(\mathrm{Ma}=0.7, \alpha=3.5^{\circ}\right.$ and $\left.\operatorname{Re}=2.6 \cdot 10^{6}\right)$. The effectiveness of the SCBs for different spanwise spacings (ranging from $20 \% \mathrm{c}$ to $30 \% \mathrm{c}$ ) was verified using two optical techniques: schlieren visualization and particle image velocimetry (PIV). Both techniques showed the possibility of controlling buffet using such devices, resulting in a reduction of the unsteadiness present in the flow, both in terms of shock oscillation and pulsation of the separated area. A dedicated PIV investigation in a spanwisechordwise measurement plane was then conducted in order to understand the effect of the spatial distribution of the bumps, focusing on the interaction of the shockwave structures along the span. The configuration with a spacing of $\Delta \mathrm{x}_{\mathrm{SCB}}=25 \% \mathrm{c}$ demonstrated to be the most efficient in reducing the transonic buffet oscillations.
\end{abstract}

\section{Introduction}

Transonic buffet consists in the oscillation of a shockwave on the suction side of a wing, which occurs for flow conditions that comprise a specific range of angle of attack, Mach and Reynolds number combination. These oscillations could eventually result in failure of the wing due to fatigue and to the oscillation of the aerodynamic properties. Therefore, for safety reasons the flight envelope of an aircraft is limited so as not to encounter transonic buffet. The first studies on transonic buffet were performed by Ref. [1] and the phenomenon has been extensively studied in the last 30 years. However, the mechanism behind this phenomenon has not been completely understood yet. In 1990 Lee [2] was the first author to describe transonic buffet as a feedback mechanism, in which the shockwave (SW) oscillation is sustained by the occurrence of downstream travelling waves (DTWs) that move from the shock foot to the trailing edge area, and upstream travelling waves (UTWs) (which are induced by the presence of DTWs) and which, by moving in the direction of the shockwave, according to the buffet phase, allow the SW to move downstream or upstream. This model has subsequently been updated by different researchers, such as Ref. [3] who considered the UTWs capable of reaching the SW along both the suction and the pressure side. Recently a dedicated study undertaken by Ref. [4] clarified that the UTWs are acoustic waves which propagate with a velocity of $80 \mathrm{~m} / \mathrm{s}$ relative to the airfoil. A crucial contribution to the understanding of transonic 2D buffet has been given by Ref. [5] in which transonic buffet was described as the result of a modal instability, obtaining values for the buffet onset conditions which are in perfect agreement with the experimental data.

Because of the relevance of transonic buffet to safe aircraft operation in the high-subsonic regime, it is of paramount importance to be able to control or, whenever possible, suppress the phenomenon. In the last 20 years there have been many attempts to control transonic buffet as summarized in the relatively complete review article Ref. [6]. Two different strategies of control systems are available: an active and a passive control system. The first system is very attractive for its precision, but it is generally very complex to realize. An example of an active control system is given by Ref. [7], where the movement of Trailing Edge Deflectors is actuated according to the instantaneous static pressure in order to stabilize the shock oscillation.

However, in many applications a system that is as reliable as possible is preferred, for which passive control systems are more attractive. The different systems are designed in such a way that the unsteadiness are either reduced or moved to higher angles of attack and/or to higher Mach numbers (moving the buffet onset boundary). These control 
systems are generally designed with the goal of damaging the feedback mechanism, accordingly, placing the control systems mainly in the shock oscillation area or at the trailing edge. An example of the latter is the use of Trailing Edge Static Deflections, used for the first time by Ref. [8]. One of its applications [9] is the use of a flap with a vertical deflection at the trailing edge (upper gurney flap), which has demonstrated to be effective in reducing transonic buffet unsteadiness. Another possibility, widely present in literature, is the use of a vortex generator [8] which energizes the boundary layer, promoting attached flow and, therefore, inhibiting the shock induced separation.

A common system used for the control of transonic and supersonic shock interactions, is achieved by using a shock control bump (SCB), a type of device that has been extensively described in the review Ref. [10]. It is generally placed on the suction side of an airfoil and it consists in a ramp and a tail oriented in the direction of the flow. The working principle of this kind of control system is associated with the formation of a $\lambda$-shock structure instead of the traditional quasi-normal shockwave. Ref. [11,12] have shown that 3D shock control bumps are more efficient than 2D SCBs (which do not have any variation along the span), particularly in off-design conditions thanks to the streamwise vortices developing from the tail of the bump. The vortex development, together with the formation of the $\lambda$-shock structure, are the two main flow structures associated with a 3D SCBs. Nowadays the use of 3D SCBs is widely present in literature and first appeared in Ref. [13] and later in Ref. [14]. Among the different shapes of SCBs one of the most common is the narrow wedge SCB, which is accurately described in Ref. [11,15]. The geometry of a wedge bump is characterized by a flat ramp, crest and tail and by angular side flanks. Because of its properties this SCB can be used as a "smart" vortex generator and applied to transonic buffet control as shown by Ref. [16]. Despite this, the usefulness of 3D SCBs for controlling transonic buffet is not univocal as observed by Ref. [17].

To achieve an efficient control system the location of the SCBs on the airfoil should be selected carefully, with the necessity of having the quasi-normal shockwave on the crest of the bump in on-design conditions. In this way no re-expansion or second shockwave would occur, as described by Ref. [10]. The use of 3D SCBs also cause a curvature of the oblique shockwave around the bump, a phenomenon that was described by Ref. [12]. When an array of bumps is used, overlapping shock structures appear, with the effectiveness of this interaction highly dependent on the relative spacing between the bumps.

With this study the authors aim to achieve a deeper understanding of the influence and effectiveness of 3D SCBs on transonic buffet, and to reach this goal an experimental study was carried out, using two optical diagnostic techniques. With schlieren visualization a qualitative description of the shock dynamics is given, whereas a more detailed quantification of the velocity field is achieved with particle image velocimetry (PIV). In addition, to the best of the authors' knowledge, no detailed study on the spacing between bumps has been carried out, except for the study of Ref. [18] done for a swept wing. Therefore, this aspect has also been addressed with a detailed PIV study on a plane parallel to the suction side of the airfoil for different spacing between the bumps.

\section{Experimental Investigation}

\section{A. Facility and airfoil}

The experiments were carried out in the TST-27, a transonic-supersonic blowdown wind tunnel of TU Delft, with a test section $25 \mathrm{~cm}$ high and $28 \mathrm{~cm}$ wide. In this work the wind tunnel has been operated for transonic conditions at a total pressure of $\mathrm{p}_{0}=2$ bars and a total temperature of $\mathrm{T}_{0}=288 \mathrm{k}$. The flow conditions are summarized in Table 1

Two models have been used in the different experiments. They are both based on the OAT15A airfoil, which is a supercritical airfoil, widely used for transonic buffet research [3]. Both models have a chord (c) of $10 \mathrm{~cm}$, but they differ in span as summarized in Table 2. The first, having a span of $28 \mathrm{~cm}$, is clamped on both sides of the wind tunnel (Fig. 1 (left)) and referred to as the "horizontal" airfoil. The second model, referred to as the "vertical" airfoil, has a span of $20 \mathrm{~cm}$ and in operational conditions is oriented in the wind tunnel vertically, with the suction side parallel to the side wall of the tunnel. This model, which is attached to one of the side walls of the wind tunnel by means of pylons, as shown in Fig. 1 (right), has previously been used in the study reported in Ref. [19]. For both models, a transition trip was applied at $7 \%$ of the chord in order to ensure a fully turbulent boundary layer. All the tests have been performed for $M a=0.7$ and $\alpha=3.5^{\circ}$ and $\operatorname{Re}_{c}=2.6 \cdot 10^{6}$.

Table 1 Flow conditions.

\begin{tabular}{l|l|l}
\hline Parameter & Symbol & Value \\
\hline Free stream Mach number & $\mathrm{Ma}_{\infty}$ & 0.7 \\
Free stream velocity & $\mathrm{U}_{\infty}$ & $225 \mathrm{~m} / \mathrm{s}$ \\
Total temperature & $\mathrm{T}_{0}$ & $288 \mathrm{~K}$ \\
Total pressure & $\mathrm{p}_{0}$ & $2 \mathrm{bar}$
\end{tabular}



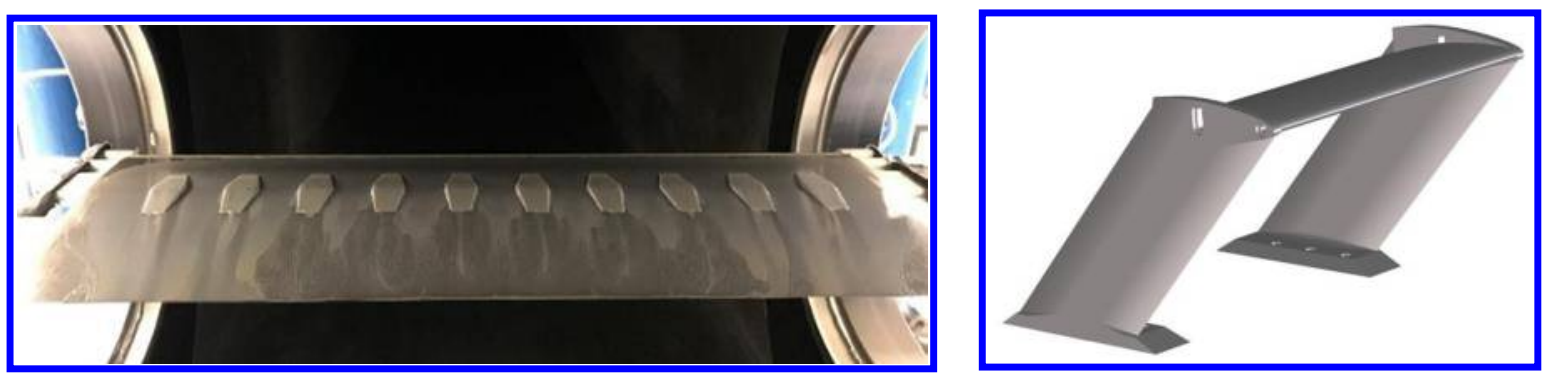

Fig. 1 SCBs (spacing 25\%c) mounted on the "horizontal" OAT15A airfoil with oil flow visualization $\left(\mathrm{Ma}=0.7, \alpha=3.5^{\circ}\right)$ on the left and assembly of the "vertical" airfoil mounted on its pylons on the right.

\section{B. Shock control bump design}

The SCBs used in this study have been produced with a 3D printer in plastic material (PLA) with an accuracy of $20 \mu \mathrm{m}$. The use of SCBs in SWBLI in literature is widespread, but nevertheless, there is not much information regarding their design and location. For the current investigation the SCB design has been based on Ref. [11,14].

The geometry and dimensions of the realized SCB are shown in Fig. 2, a length of $28 \mathrm{~mm}$ and a maximum height of $0.9 \mathrm{~mm}$ have been chosen. The height of the bumps (including the double sided adhesive tape with which the bumps are attached to the airfoil) has been selected equal to the local height of the boundary layer. The crest of the bump has been centred at the average shock location for the most developed buffet condition $\left(M a=0.7\right.$ and $\alpha=3.5^{\circ}$ ), which corresponds to $45 \%$ of the chord. It is expected that for this buffet condition the oscillating shockwave will acquire the familiar $\lambda$ structure, stabilizing the shock oscillation.

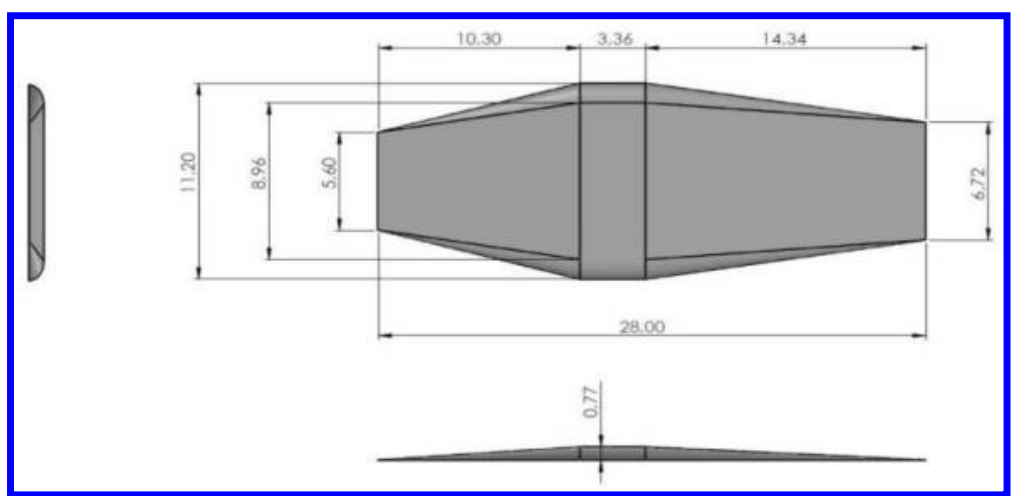

Fig. 2 Geometry of a shock control bump with dimensions in $\mathbf{m m}$.

The spanwise spacing between the bumps is a crucial parameter, as it determines if the interaction among the different shockwave structures in correspondence of the bumps will be detrimental or beneficial. However, since there is no evidence of a study like this in literature, an investigation of this aspect has been addressed, different spacings, namely: 20, 22.5, 25, 27.5, 30\%c (selected in the range of values reported in other publications [12,15]).

Figure 1 (left) shows the OAT15A with bumps after a run at $\mathrm{Ma}=0.7$ and $\alpha=3.5^{\circ}$ and provides an oil flow visualization for a spacing between the bumps of $25 \mathrm{~mm}(25 \% \mathrm{c})$.

Table 2 Model parameters.

\begin{tabular}{l|l|l}
\hline Parameter & Symbol & Value \\
\hline Chord & $\mathrm{c}$ & $10 \mathrm{~cm}$ \\
Span ("horizontal model") & $\mathrm{b}_{1}$ & $28 \mathrm{~cm}$ \\
Span ("vertical model") & $\mathrm{b}_{2}$ & $20 \mathrm{~cm}$ \\
Angle of attack & $\alpha$ & $3.5^{\circ}$ \\
Reynolds based on chord & $\operatorname{Re}_{\mathrm{c}}$ & $2.6 \cdot 10^{6}$
\end{tabular}




\section{Experimental set-up}

In this study two optical techniques have been used: schlieren and particle image velocimetry (PIV).

High speed schlieren was used to visualize the shock dynamics using a high speed camera (Photron Fastcam SA1.1) with an acquisition frequency of $5 \mathrm{kHz}$, which is high enough to resolve the shock oscillation motion that occurs with a characteristic frequency of $160 \mathrm{~Hz}$ [20]. With this technique the different spacing between the bumps was analysed for the first model (airfoil clamped on the side-walls of the wind tunnel).

Subsequently, a quantitative study of the velocity field was performed with PIV and in this case two different experiments were performed.

In the first experiment high speed PIV was carried out for the "horizontal" airfoil. An acquisition frequency of 15 $\mathrm{kHz}$ was selected in order to temporally resolve the shock position, the vortex shedding and the propagation of upstream travelling pressure waves. To achieve this acquisition frequency, the sensor of each camera (operating in double pulse mode with a time separation $d t=3 \mu s$ ) was cropped to a resolution of $576 \times 320$ pixels. Despite this, a good spatial resolution was still achieved by simultaneously using three cameras in planar mode. A sketch of the experimental set-up is shown in Fig.3 (left), while Fig. 3 (right) indicates the different FOVs (developing from 26\% to $100 \% \mathrm{c}$ ). All these measurements were performed in the chordwise-vertical plane at mid span, in correspondence of the central bump.

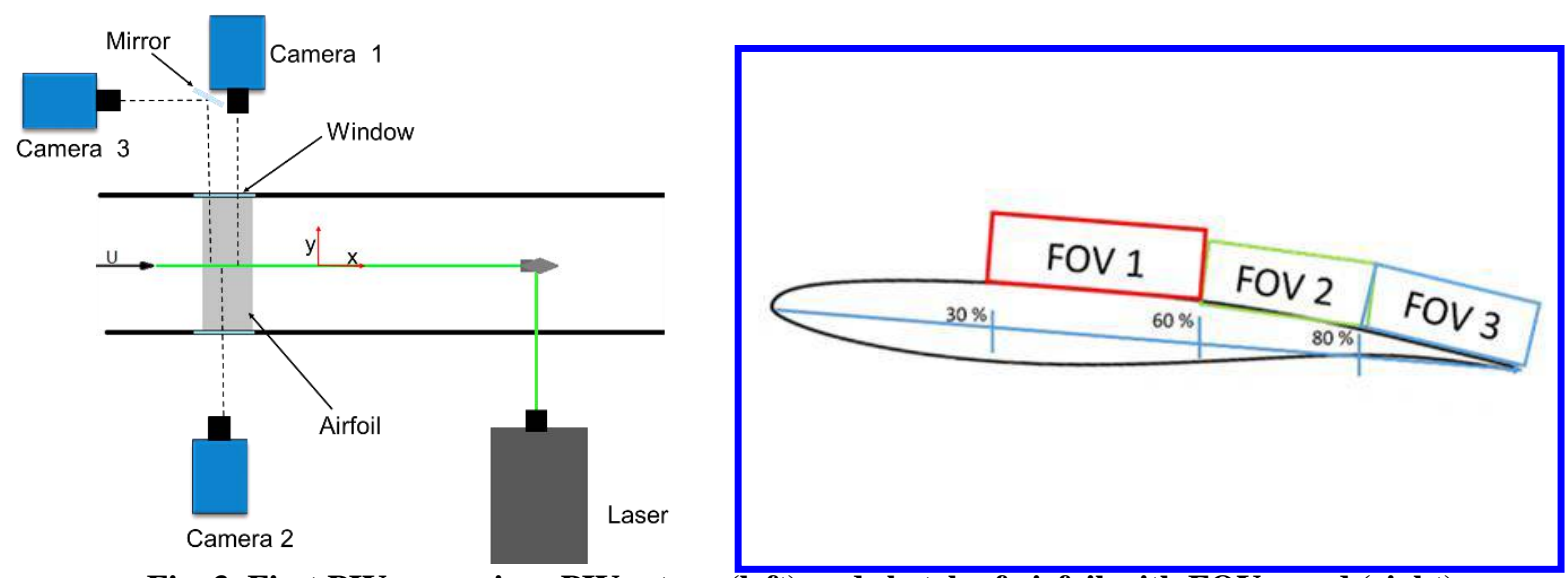

Fig. 3 First PIV campaign: PIV set-up (left) and sketch of airfoil with FOVs used (right).

In order to appreciate the spanwise distribution of the shockwave a further PIV experiment was completed in a spanwise-chordwise oriented plane on the "vertical" model, whose orientation in the test section allows a direct access from the side window towards the suction side of the airfoil (Fig. 4 (left)). One high speed camera (again a Photron Fastcam SA1.1) in planar configuration has been used, with a selected frequency of acquisition of $5 \mathrm{kHz}$ (sensor of the camera cropped to $1024 \times 640$ pixels) and a FOV extending from $15 \% \mathrm{c}$ to the trailing edge. As illustrated in Fig. 4 (right), the plane of measurement is located at a distance of $\mathrm{z}-\mathrm{Z}_{\mathrm{w}}=0.5 \mathrm{~cm}$ from the thickest point of the airfoil (where $\mathrm{Z}_{\mathrm{w}}$ is the $\mathrm{z}$ coordinate of the surface of the airfoil in correspondence of the thickest point).

For all the PIV tests, a dual cavity high speed laser (Nd:YAG) has been used in order to illuminate the seeding particles, which in this study are droplets of DEHS (Di-Ethyl-Hexyl-Sebacat). The synchronization between the cameras and the laser was achieved through a synchronization box (LaVision High Speed Controller).

\section{Processing procedures}

The schlieren images were acquired with the camera software and then processed with Matlab. Differently, for the PIV measurements, the image acquisition was performed with the LaVision software DAVIS. The same software has been used for image pre-processing and processing (computation of velocity field by means of a cross-correlation procedure) while further processing was carried out in Matlab. For the PIV image processing, first a Butterworth filter was applied to reduce the laser reflections on the airfoil and on the SCBs, later the cross-correlation was computed with a multi-pass approach. For the campaign on the horizontal airfoil, one pass with a window size of $64 \times 64$ pixels and three passes with a final window size of $48 \times 48$ pixels were used, in both cases with an overlap of $75 \%$ of the window size. On the other hand, for the PIV campaign on the second (vertical) model, one pass with a window size of $48 \times 48$ pixels and three passes with a final window size of $32 \times 32$ pixels were selected, again with an overlap of 
$75 \%$. Correspondingly, the resulting spacing between the vectors is $0.47 \% \mathrm{c}$ for the first PIV campaign, and $0.67 \% \mathrm{c}$ for the images on the vertical airfoil.

The estimation of the uncertainties in the determination of the instantaneous velocity field associated to these cross-correlation procedures, as derived in detail in Ref. [21], provide the values summarized in Table 3 for each of the PIV campaigns.
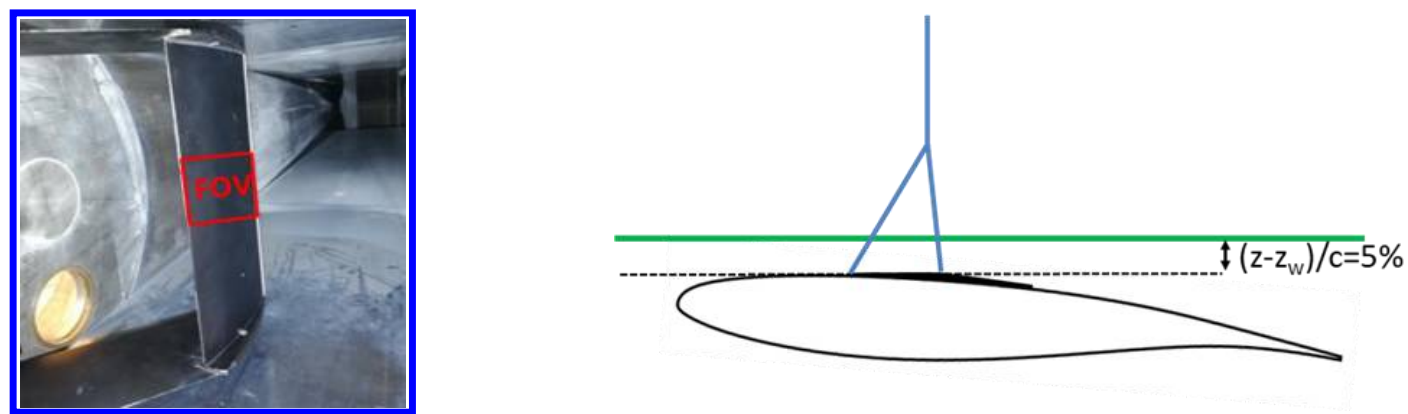

Fig. 4 Second PIV experiment: "vertical" airfoil mounted in the wind tunnel with relative FOV (left) and sketch of the airfoil with the plane of measurement in green and $\lambda$-shock structure in blue (right).

Table 3 PIV parameters.

\begin{tabular}{l|l|l}
\hline Parameter & First PIV campaign & Second PIV campaign \\
\hline Model orientation & horizontal & vertical \\
Acquisition frequency & $15 \mathrm{kHz}$ & $4.65 \mathrm{kHz}$ \\
Number of images & 15500 & 4365 \\
Final resolution & $1600 \times 320 \mathrm{pix}$ & $1024 \times 640 \mathrm{pix}$ \\
Final window size & $48 \times 48 \mathrm{pix}$ & $32 \times 32 \mathrm{pix}$ \\
Window overlap & $75 \%$ & $75 \%$ \\
Vector spacing & $0.47 \% \mathrm{c}$ & $0.67 \% \mathrm{c}$ \\
Cross-correlation uncertainty & $<3 \mathrm{~m} / \mathrm{s}$ & $<10 \mathrm{~m} / \mathrm{s}$
\end{tabular}

\section{Shock dynamics}

In Fig. 5 instantaneous schlieren images are shown for both the clean airfoil (left) and the SCB configuration with a spacing $\left(\Delta \mathrm{x}_{\mathrm{SCB}}\right)$ of $25 \% \mathrm{c}($ right $)$. In the latter, two oblique lines appear in the image; going from left to right, the first is associated with a Mach line caused by the transition trip that is located at $7 \%$ of the chord (in order to ensure a turbulent boundary layer), while the second is an oblique shockwave created at the leading edge of the bumps (the latter is indicated in blue) followed by a quasi-normal shockwave. In correspondence of the interactions of the the quasi-normal shockwave with the Mach line and with the oblique SW, two slip lines are present. Downstream of the shockwave, the presence of a separated area can also be visualized, thanks to the knife edge horizontal orientation. For the clean airfoil (Fig. 5, left), the lambda shockwave is substituted by a quasi-normal shockwave.
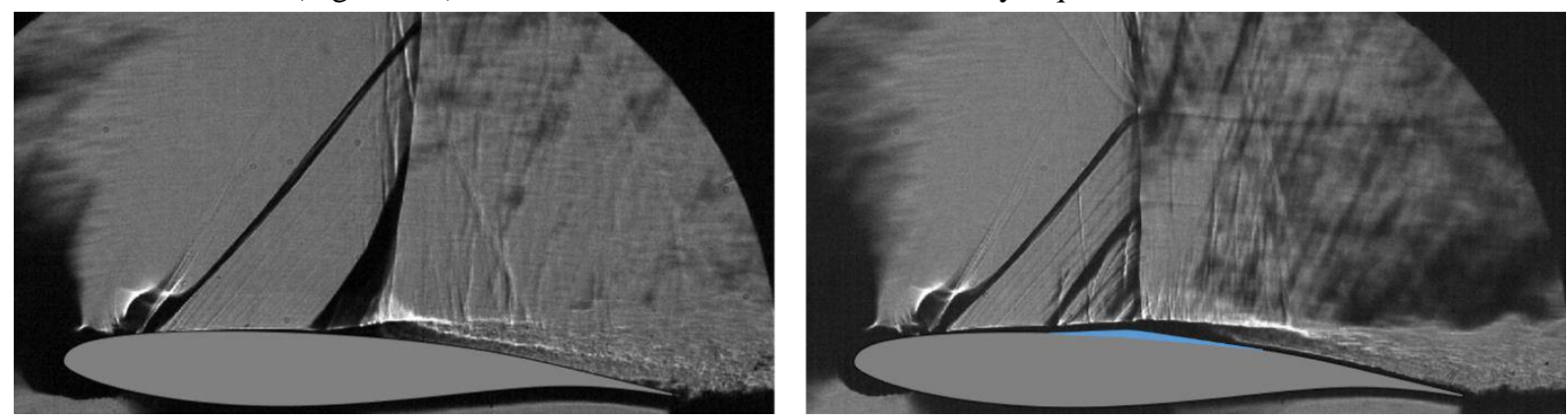

Fig. 5 Example of a schlieren image of the OAT15A at $\mathrm{Ma}=0.7, \alpha=3.5^{\circ}$ with bumps (right) and without (left). 
In this study the main flow features have been further investigated using PIV measurements. In Fig. 6 instantaneous PIV images in the chordwise-vertical plane are shown, for both the clean (left) and the SCB configuration with $\Delta \mathrm{x}_{\mathrm{SCB}}=25 \% \mathrm{c}$ (right). The two images have both been captured during the upstream travel of the shockwave. For the clean configuration only a moving oblique shockwave is observed at $35 \% \mathrm{c}$. Differently, for the controlled configuration (as already commented for the schlieren images) there is both a steady oblique shockwave in correspondence to the leading edge of the bumps and a secondary moving shockwave close to $\mathrm{x}=40 \% \mathrm{c}$. In this particular buffet phase, a wide separated area is evident for both the configurations, in agreement with literature [3,9].
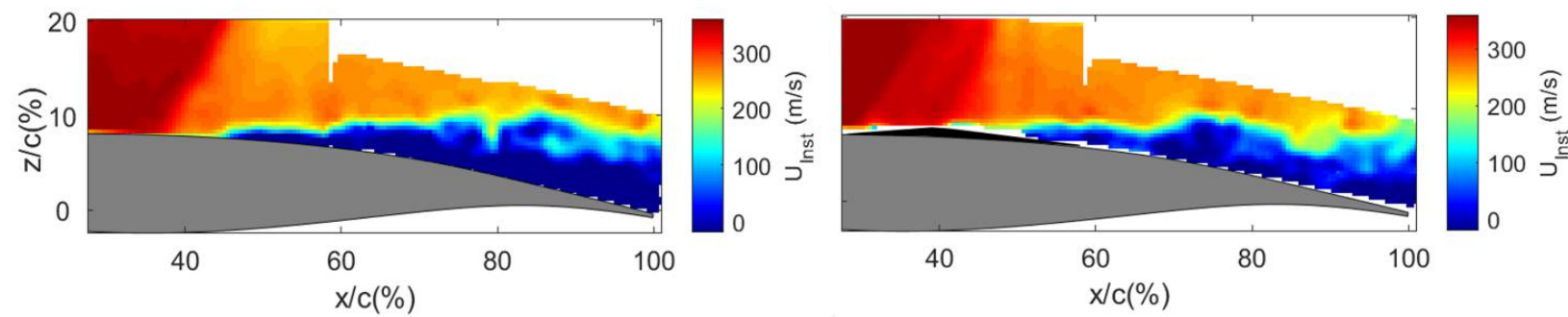

Fig. 6 Example of a PIV image of the OAT15A at Ma=0.7, $\alpha=3.5^{\circ}$ with bumps (right) and without (left).

From the schlieren recordings, the shock position has been tracked in time by evaluating the minimum intensity of luminosity in each image. In Fig. 7 (left) the probability density function $\mathrm{p}\left(\mathrm{X}_{\mathrm{Sw}} / \mathrm{c}\right)$ derived from the shock position is shown for both the clean configuration and in presence of bumps with different spacings. The result clearly illustrates that with any SCB configuration, a reduction of the area of oscillation of the SW is obtained. Furthermore, the average shock position is shifted towards more downstream positions. The best performance in terms of shockwave oscillation reduction is obtained for a bump spacing of $25 \% \mathrm{c}$, although similar results are obtained for $\Delta \mathrm{x}_{\mathrm{SCB}}=27.5 \% \mathrm{c}$ and $\Delta \mathrm{x}_{\mathrm{SCB}}=30 \% \mathrm{c}$. These observations are confirmed by the data in Table 4 , which shows the average values of the shock position and the relative standard deviations. The data confirm that for $\Delta \mathrm{x}_{\mathrm{SCB}}=25 \% \mathrm{c}$, the weakest fluctuations of the shockwave, but also the most downstream average shock position occur.
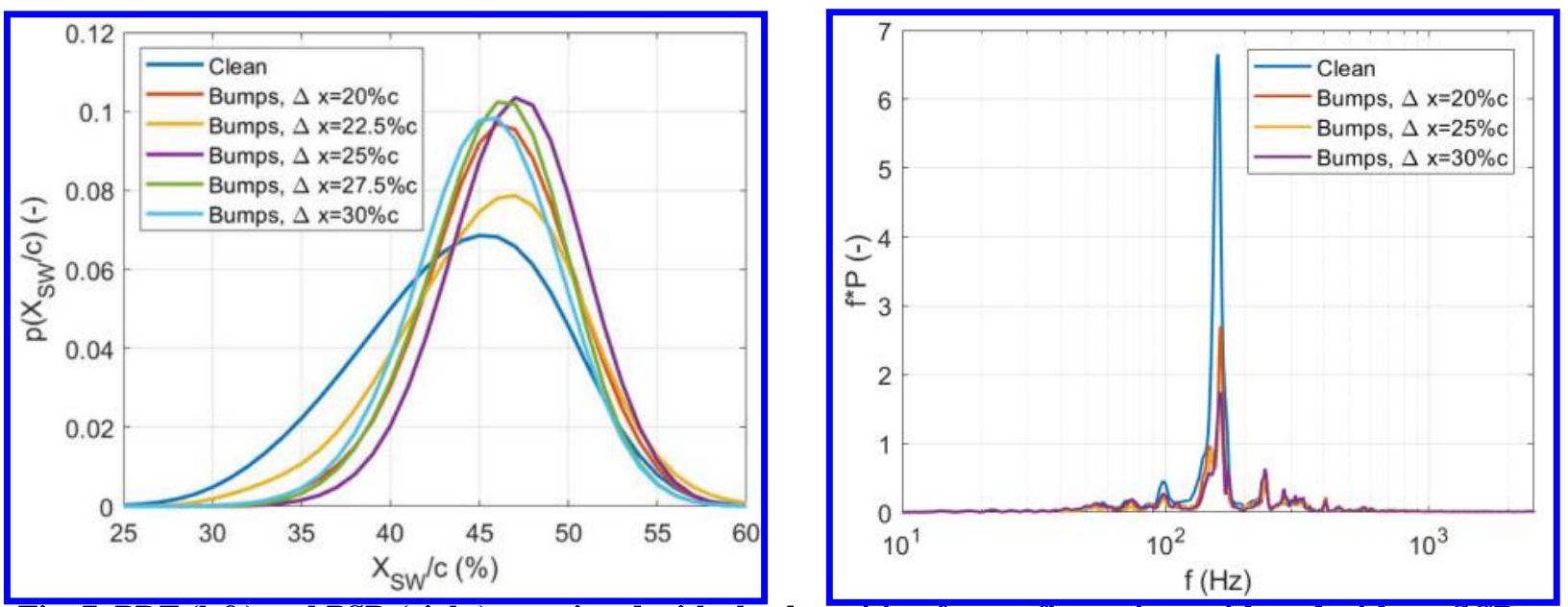

Fig. 7 PDF (left) and PSD (right) associated with shock position for configurations with and without SCBS.

The spectral content of the shock position variation is obtained in the form of a power spectral density $(\mathrm{P})$ by using the Welch method. The corresponding results are plotted in Fig. 7 (right) in terms of the pre-multiplied spectrum (f.P) for the clean configuration and for the airfoil in presence of bumps, with spacings of $20 \% \mathrm{c}, 25 \% \mathrm{c}$ and $30 \% \mathrm{c}$. The image clarifies that the buffet characteristic frequency is not affected by the use of SCBs, with all the configurations having a main peak at $160 \mathrm{~Hz}$, which is in good agreement in terms of Strouhal number (St=0.07) with Ref. [3]. However, all the SCB configurations achieve a relevant reduction of the main peak of $\mathrm{f} \cdot \mathrm{P}$, although the most important reduction is obtained for $\Delta \mathrm{x}_{\mathrm{SCB}}=25 \% \mathrm{c}$. The values of $\mathrm{f} \cdot \mathrm{P}$ at $160 \mathrm{~Hz}$ are summarized in Table 4 for all the tested configurations.

By computing the maximum of the gradient of velocity for the PIV images, the shock position has been tracked for the velocity data as well. The corresponding power spectral density (Fig. 8), confirms the result shown in Fig. 7 
(right), with a reduction of more than $50 \%$ of the main peak at $160 \mathrm{~Hz}$ when the SCBs are used. Similar results are obtained for both $\Delta \mathrm{x}_{\mathrm{SCB}}=25 \% \mathrm{c}$ and $\Delta \mathrm{x}_{\mathrm{SCB}}=30 \% \mathrm{c}$. Therefore, the results are in good agreement with the schlieren data.

Table 4 Comparison of shock position properties for different SCB spacings from schlieren data.

\begin{tabular}{l|l|l|l}
\hline & $\mathbf{X}_{\text {shock }}(\boldsymbol{\%})$ & $\mathbf{S T D}\left(\mathbf{X}_{\text {shock }}\right)(\boldsymbol{\%})$ & $\mathbf{f} \cdot \mathbf{P}(\mathbf{f}=\mathbf{1 6 0} \mathbf{H z})(-)$ \\
\hline Clean configuration & 43.78 & 5.28 & 6.65 \\
$\Delta \mathrm{x}_{S C B}=20 \% \mathrm{c}$ & 46.07 & 3.73 & 2.71 \\
$\Delta \mathrm{x}_{\mathrm{SCB}}=22.5 \% \mathrm{c}$ & 45.54 & 3.98 & 3.02 \\
$\Delta \mathrm{x}_{\mathrm{SCB}}=25 \% \mathrm{c}$ & 46.97 & 3.35 & 1.53 \\
$\Delta \mathrm{x}_{\mathrm{SCB}}=27.5 \% \mathrm{c}$ & 45.91 & 3.39 & 2.00 \\
$\Delta \mathrm{x}_{\mathrm{SCB}}=30 \% \mathrm{c}$ & 45.43 & 3.58 & 1.75
\end{tabular}

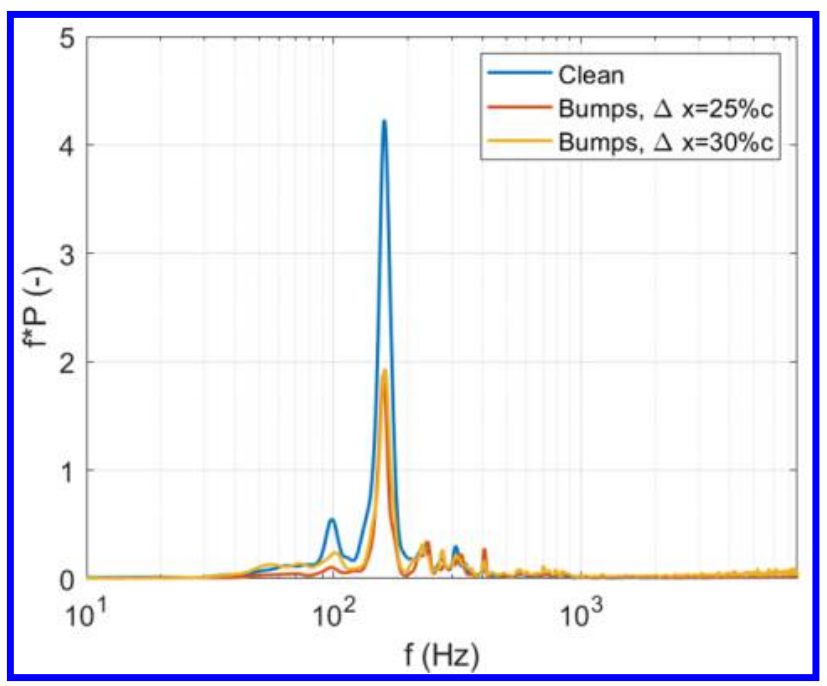

Fig. 8 Spectral analysis of PIV shock position for different configurations.

\section{Flow field analysis}

\section{A. Buffet cycle in presence of 3D SCBs}

In order to gain further insight on the effect of 3D SCBs on the buffet cycle, PIV instantaneous images are shown in two particular stages of the buffet cycle, corresponding to the most upstream and the most downstream position of the shock oscillation, respectively. The images (Figs. 9, 10) are shown for the $\Delta \mathrm{x}_{\mathrm{SCB}}=25 \%$ c configuration only.

The snapshot in Fig. 9 (left) shows the velocity field in the stage in which the shock approaches the most upstream position. In this case the moving shockwave is located slightly upstream (36\%c) of the crest of the SCB. The flow field also shows the presence of a separated area, whose extent is significantly smaller than the region present in Fig. 6 (right). In Fig. 9 (right) it is possible to visualize the instantaneous spanwise organization of the velocity field. This snapshot has been acquired in a buffet phase similar to the phase depicted in Fig. 9 (left) (using the data on the vertical airfoil). Figure 9 (right) shows that the oblique shockwaves originate at the leading edge of the SCBs, curve around the bumps, with the SW structures eventually interacting with each other. Downstream of the curved shockwave, the quasi-normal moving shockwave displays a very coherent distribution along the span of the airfoil.

Figure 10 (left) shows the instantaneous velocity field with the quasi-normal SW in proximity of the most downstream position (close to 50\%c). Even in this case the separated area extent is quite limited. As for the clean airfoil [3,9], in presence of SCBs the separated area reaches its maximum extent during its upstream travel (see Fig. 6) and its lowest extent during its downstream travel (although this situation is not shown in this study). 

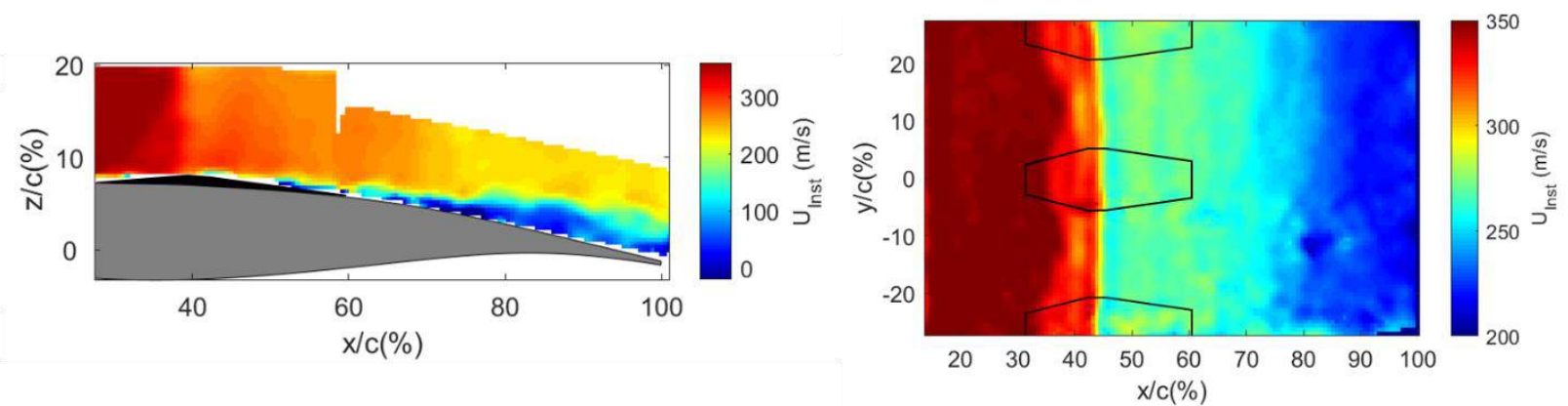

Fig. 9 Uncorrelated instantaneous horizontal velocity fields in presence of $\mathrm{SCBs}\left(\Delta \mathrm{xsCB}_{\mathrm{S}}=25 \% \mathrm{c}\right)$ with the $\mathrm{SW}$ in its most upstream position. On the left the velocity field is shown in the chordwise-vertical plane, while on the right another snapshot is displayed in the chordwise-spanwise plane together with the outline of the bumps.

By looking at the chordwise-spanwise distribution of velocity, the presence of the different curved steady shockwaves originating at the leading edge of the bumps is still evident. The supersonic area is terminated once again with a $2 \mathrm{D}$ quasi-normal shockwave.

Despite this observed spanwise coherence, the separated area is not expected to be 2D, as suggested by the oil flow visualization in Fig. 1 (left). From this image, it seems that the separated areas at the back of the different SCBs merge with each other from only 70\%c onwards. This information cannot be extracted from the PIV data from the vertical airfoil, since the measurement plane was detached from the surface of the airfoil, and located just above the separated area region (see Fig. 4, right).
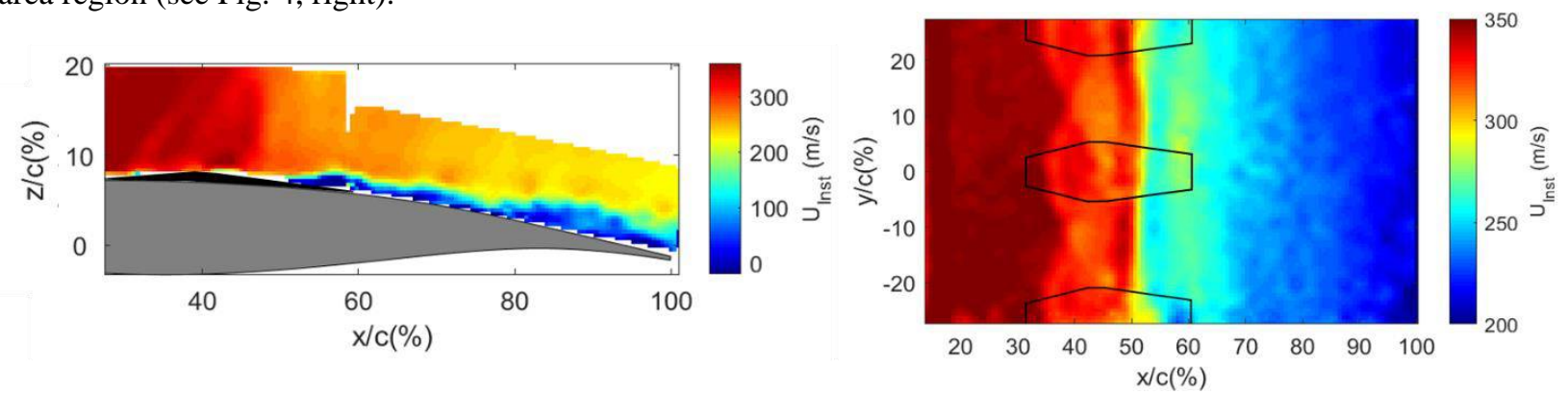

Fig. 10 Uncorrelated instantaneous horizontal velocity fields in presence of $\mathrm{SCBs}\left(\Delta \mathrm{x}_{\mathrm{SCB}}=25 \% \mathrm{c}\right)$ with the $\mathrm{SW}$ in its most downstream position. On the left the velocity field is shown in the chordwise-vertical plane, while on the right another snapshot is displayed in the chordwise-spanwise plane together with the outline of the bumps.

\section{B. Main statistics of the velocity field}

A first quantitative evaluation of the velocity field is provided by the distribution of the average horizontal velocity component for both the clean airfoil and the airfoil in presence of the best performing SCB configuration (Fig. 11). The main flow structures are easily observed in both the configurations. For the uncontrolled airfoil, the mean SW location is not easily identifiable because of the SW oscillation, which results in a gradual compression rather than a sharp interface. In contrast, in presence of control devices, an oblique SW at the leading edge of the SCB is clearly observed. From this comparison no important difference is observed in terms of extent of the separated area, however, a wider shear layer (approximated by the area included among the two contour lines) seems to be present for the clean airfoil.
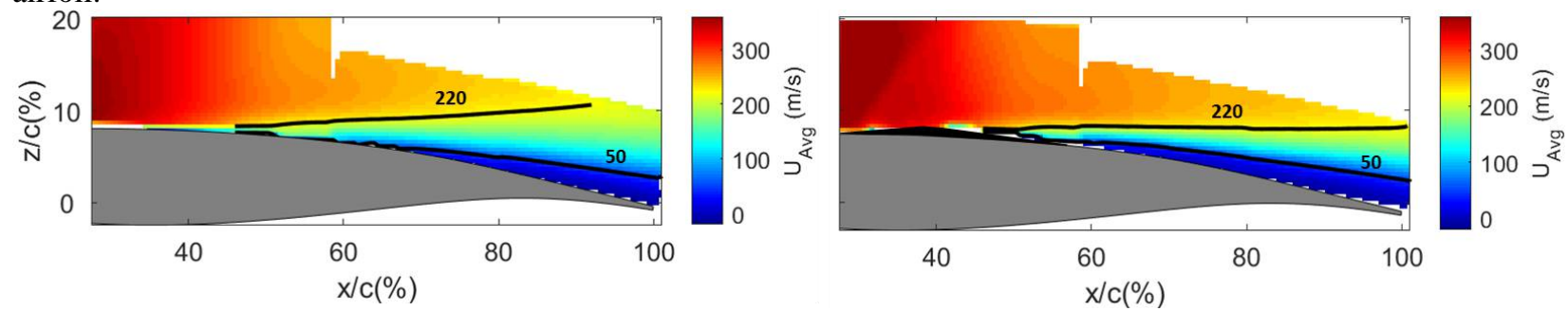

Fig. 11 Average horizontal velocity with contour lines at 220 and $50 \mathrm{~m} / \mathrm{s}$. On the left the velocity field is shown for the for the clean airfoil, while on the right for the airfoil in presence of SCBs with $\Delta x_{S C B}=25 \% c$. 
In order to highlight the unsteadiness present in the flow field, Fig. 12 shows the standard deviation of the horizontal component of the velocity field for the same configurations shown in Fig. 11. In terms of SW position, the results are in good agreement with those presented in the previous section, with a smaller range of oscillation of the SW for the controlled case. These ranges are in accordance with those shown in Fig. 7 (left), and furthermore confirm the stabilizing effect obtained with the $\lambda$-shape of the SW. In proximity of $\mathrm{z} / \mathrm{c}=20 \%$ and $\mathrm{x} / \mathrm{c}=40 \%$ there is an increase of the standard deviation values for the SCB configuration. This growth is associated with the moving shockwave also travelling (at that location) upstream of the steady oblique shockwave when reaching its most upstream position. The distribution of standard deviation downstream of the shockwave, confirms a reduction of the fluctuation of both the separated area and of the shear layer in presence of SCBs.
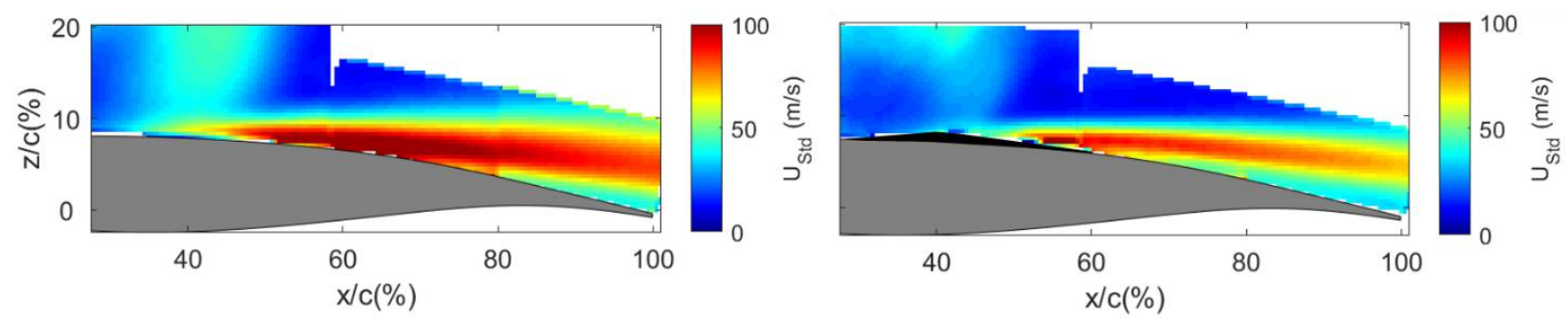

Fig. 12 Standard deviation of the horizontal velocity component for the clean airfoil (left) and in presence of SCBs with $\Delta \mathrm{x}_{S C B}=25 \% \mathrm{c}$ (right).

\section{Separated and shear layer area behaviour}

The extent of the separated area and of the shear layer has been extracted from the average flow field to further asses their behavior. The separated area extent has been defined as the percentage of velocity vectors (downstream of $\mathrm{x} / \mathrm{c}=60 \%$ ) with a velocity lower than $50 \mathrm{~m} / \mathrm{s}$, while for the shear layer with a velocity included between 50 and 220 $\mathrm{m} / \mathrm{s}$.

The results are shown in Table 5. Regarding the separated area, there is a slight increase of its extent for the best behaving SCB configuration compared to the clean configuration (20\% against $17 \%$ of vectors with a velocity lower than $50 \mathrm{~m} / \mathrm{s}$ ). This result was to be expected, considering the PIV measurement plane is passing in the symmetry plane of the central bump and therefore it is in its wake. Differently, for $\Delta \mathrm{x}_{\mathrm{SCB}}=25 \% \mathrm{c}$ there is an important reduction of the extent of the shear layer (from 54 to $37 \%$ of the total vectors). This beneficial effect could result from the vortical structures developing from the tail of the bumps, which is described accurately in Ref. [11,12]. Furthermore, in presence of bumps the simultaneous presence of an oblique shockwave and of a quasi-normal shockwave is less dissipative compared to the single shockwave present for the clean configuration. On the other hand, the SCB configuration with $\Delta \mathrm{x}_{\mathrm{SCB}}=30 \% \mathrm{c}$ is characterized by both a wider separated area and shear layer in comparison to $\Delta \mathrm{x}_{\mathrm{SCB}}=25 \%$ c, confirming the importance of the spacing of the bumps and the corresponding flow structures.

Table 5 Separated area and shear layer extent.

\begin{tabular}{l|l|l}
\hline Parameter & $\begin{array}{l}\text { Vectors belonging to } \\
\text { separated area }(\%)\end{array}$ & $\begin{array}{l}\text { Vectors belonging to } \\
\text { shear layer area }(\%)\end{array}$ \\
\hline Clean configuration & 17 & 54 \\
$\Delta \mathrm{x}_{\mathrm{SCB}}=25 \% \mathrm{c}$ & 20 & 37 \\
$\Delta \mathrm{x}_{\mathrm{SCB}}=30 \% \mathrm{c}$ & 23 & 50
\end{tabular}

In Fig. 13 profiles of the horizontal velocity components are plotted for $\mathrm{x} / \mathrm{c}=60 \%, \mathrm{x} / \mathrm{c}=80 \%$ and $\mathrm{x} / \mathrm{c}=100 \%$. All the profiles are shown with respect to the local height of the surface $\mathrm{z}_{\mathrm{w}}(\mathrm{x} / \mathrm{c})$ therefore, the local distance from the surface of the airfoil is always indicated on the $\mathrm{y}$-axis $\left(\mathrm{z}-\mathrm{Z}_{\mathrm{w}}(\mathrm{x} / \mathrm{c})\right) / \mathrm{c}$. The $\Delta \mathrm{x}_{\mathrm{SCB}}=25 \% \mathrm{c}$ profiles are all on the left of the profiles for the clean case in proximity of the surface of the airfoil (in the separated area) and on the right after the inflection points of the former. The shape of the profile once again suggests a thinning of the shear layer for the SCB configuration with a spacing of $25 \% \mathrm{c}$. On the contrary, this effect is not observed for the second spacing tested, with its profile almost overlapping the profile of the clean configuration for $\left(\mathrm{z}-\mathrm{Z}_{\mathrm{w}}(\mathrm{x} / \mathrm{c})\right) / \mathrm{c}>10 \% \mathrm{c}$. 

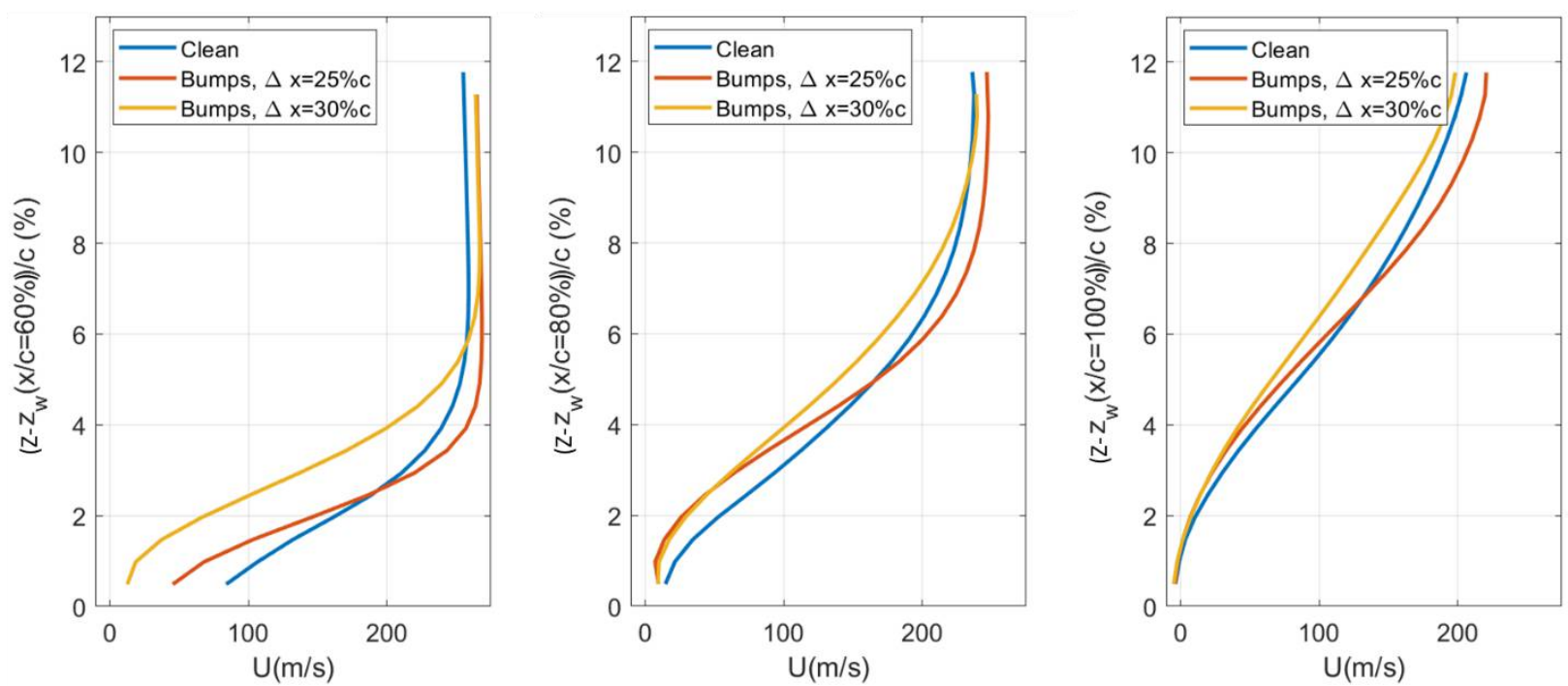

Fig. 13 Horizontal velocity profile at $x / c=60 \%$ (left), $x / c=80 \%$ (centre) and $x / c=100 \%$ (right) for the three configurations tested.

In Fig. 14, the profiles of the standard deviation of the horizontal velocity are shown for the same configurations. The graphs clarify that with a spacing of $25 \% \mathrm{c}$ the fluctuations in the separated area are always reduced when compared to the clean configuration. On the other hand, the $\Delta \mathrm{x}_{\mathrm{SCB}}=30 \% \mathrm{c}$ allows a reduction of the fluctuations for $\mathrm{x} / \mathrm{c}=80 \%$ and $\mathrm{x} / \mathrm{c}=100 \%$ but an increase just downstream of the SW oscillating range, for $\mathrm{x} / \mathrm{c}=60 \%$.
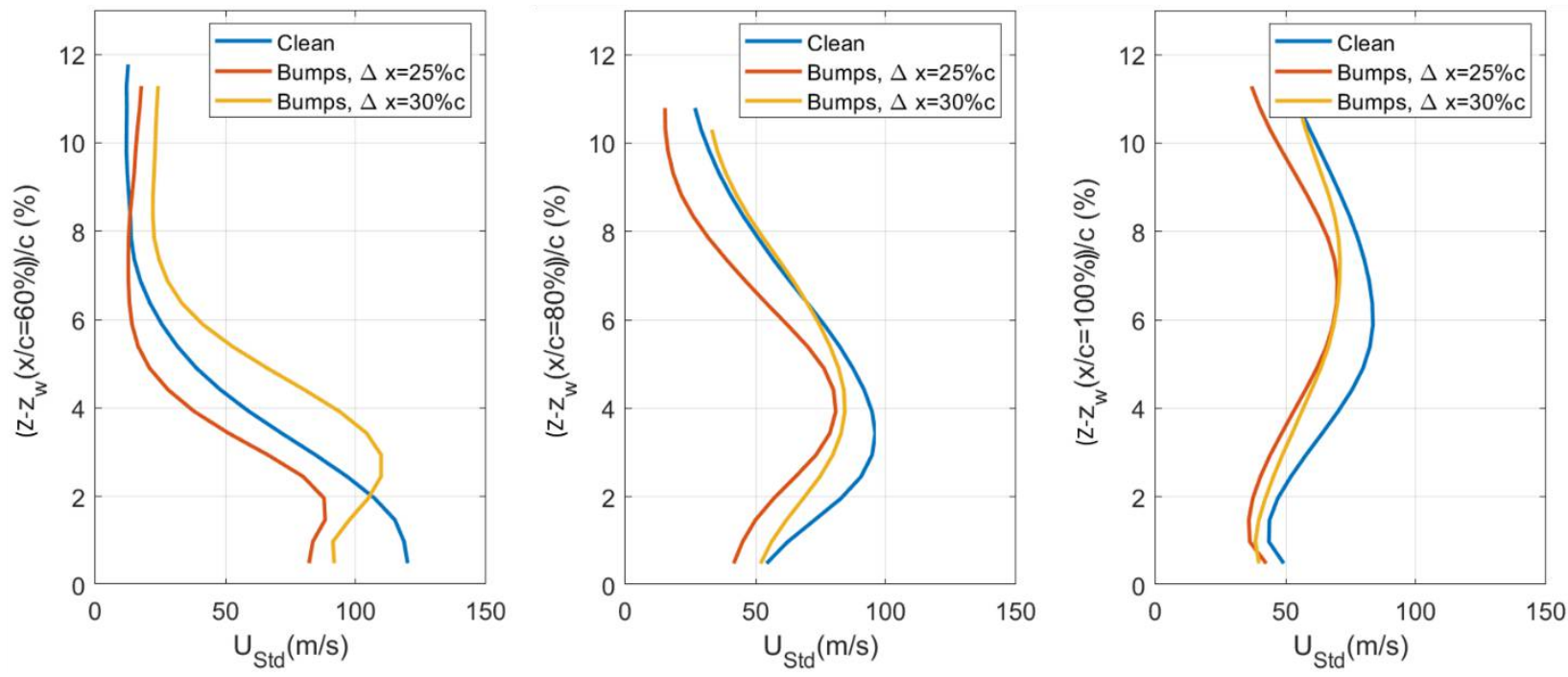

Fig. 14 Profile of STD of horizontal velocity at $x / c=60 \%$ (left), $x / c=80 \%$ (centre) and $x / c=100 \%$ (right) for the three configurations tested.

By tracking the velocity fluctuations in the middle of the high fluctuations region in the separated area $(\mathrm{x} / \mathrm{c}=80 \%$ and $\mathrm{z} / \mathrm{c}=7 \%$, see Fig. 12), the relative spectral content has been derived for the different configurations studied. The results are shown in Fig. 15 and confirm that the separated area is clearly oscillating with the same frequency of the shock oscillation $(160 \mathrm{~Hz})$. By comparing the difference in behavior, it is confirmed that these oscillations are less significant in presence of SCBs (as also clear from the standard deviation plot in Fig. 12), with the performance optimized for $\Delta \mathrm{x}_{\mathrm{SCB}}=25 \% \mathrm{c}$. The reduction of the buffet main peak for the separated area in presence of SCBs is very similar to the one observed for the shock position in Fig. 8, confirming once again that the SW oscillation and the pulsation of the separated area are closely related. 


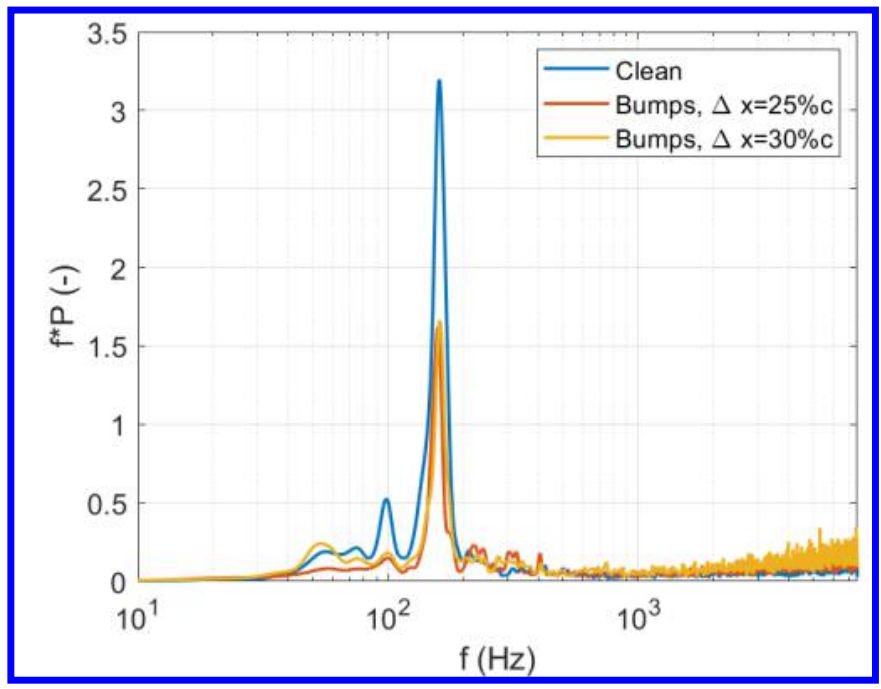

Fig. 15 Spectral analysis of $U\left(x_{0}, z_{0}, t\right)$ in a point in the separated area $\left(\mathrm{x}_{0} / \mathrm{c}=80 \%, \mathrm{z}_{0} / \mathrm{c}=7 \%\right)$.

\section{Effect of SCB spacing on the average flow field}

To better understand the difference in behavior between the bump configuration with spacing $\Delta \mathrm{x}_{\mathrm{SCB}}=25 \% \mathrm{c}$ and $\Delta \mathrm{x}_{\mathrm{SCB}}=30 \% \mathrm{c}$, the relative average velocity fields in the spanwise-chordwise plane are shown in Fig. 16. The spanwise structure of the shockwave is once again highlighted, with the presence of both a curved SW, originating from the leading edge of the bumps, and a quasi-normal SW which is located close to the crest of the bumps.

For geometric reasons the curved shockwaves developing around neighbouring bumps interact with each other at a distance $\Delta \mathrm{x}_{\mathrm{SCB}} / 2$ from the symmetry planes of the bumps. Figure 16 clarifies that the curved shockwave are still present after interacting with each other, interacting once again in correspondence of the symmetry plane of the different bumps. It is clear that the chord position of this second interaction depends strongly on the spacing $\Delta \mathrm{x}_{\mathrm{SCB}}$, with it happening much more downstream for the wider spacing. Therefore, in the case of the smaller spacing $\left(\Delta \mathrm{x}_{\mathrm{SCB}}=25 \% \mathrm{c}\right)$, the effects of the curved shockwave are not felt after $\mathrm{x} / \mathrm{c}=45 \%$. Differently for $\Delta \mathrm{x}_{\mathrm{SCB}}=30 \% \mathrm{c}$, the presence of the curved SW is observed until the most downstream position of the quasi-normal shockwave $(\mathrm{x} / \mathrm{c}=50 \%)$. This difference results in a not negligible dissimilarity in the velocity field for $35 \%<\mathrm{x} / \mathrm{c}<50 \%$. Consequently, the effect of this interaction is assumed to have an influence on the buffet oscillations and on the 2D coherence of the flow field. This observation is in agreement with Ref. [10,12], where it is stated that (although for flow conditions different than those discussed in this study) properly spaced 3D SCBs can induce a favourable quasi-2D SW along the full span.

Moreover, the distance between the SCBs directly influences the behaviour of the separated area by changing the location of interaction of the vortical structures developing from the tail of the bumps (as can be understood from the oil flow visualisation shown in Fig. 1, left).
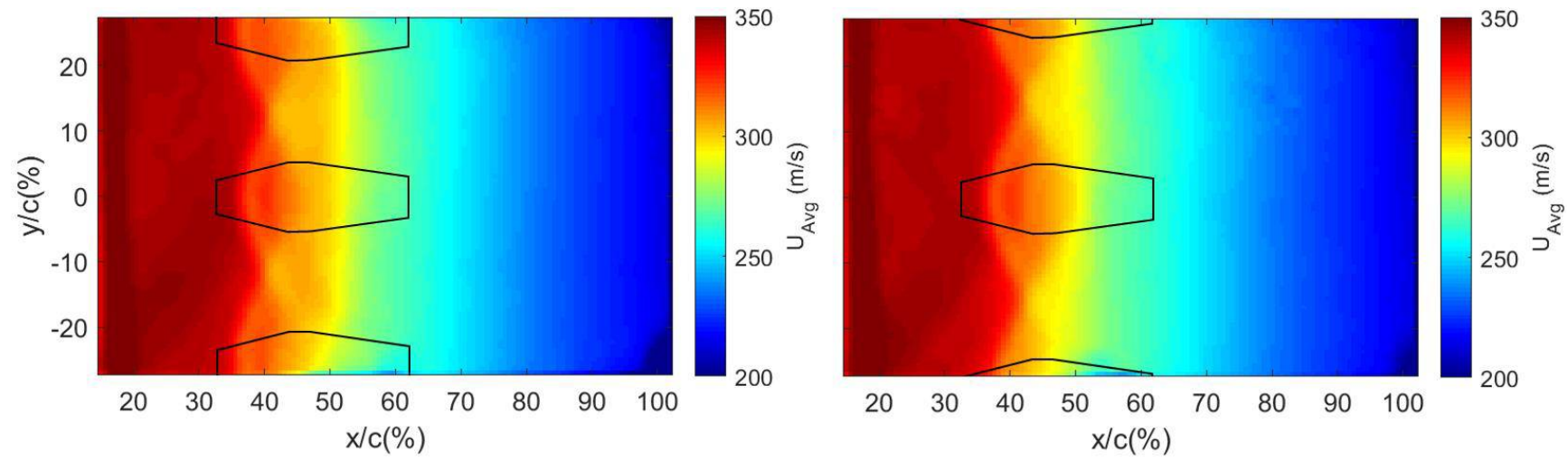

Fig. 16 Average horizontal velocity field in the spanwise-chordwise plane in presence of SCBs with $\Delta x_{S C B}=25 \% c$ (left) and with $\Delta x_{S C B}=30 \% c$ (right). In both cases the outline of the bumps is specified. 


\section{Conclusion}

The results of this study confirms that 3D SCBs are suitable devices for controlling transonic buffet. The schlieren data proved that these control devices reduce the shock oscillation (Fig. 7) by changing the structure of the SW into a $\lambda$-shape. The PIV data on the chordwise-vertical plane in Fig. 8 allow the description of the $\lambda$-structure developing on the SCBs and of the detection of the full separated area in the FOV. The corresponding detection of the SW position is in excellent agreement with the schlieren results. From a spectral point of view, the use of these devices does not affect the buffet frequency $(\mathrm{f}=160 \mathrm{~Hz})$ but clearly attenuates the contribution of the main peak. Both diagnostic techniques confirm an optimization of performance when a spacing between the bumps of $25 \%$ c is adopted. It is worth clarifying that the PIV data have been acquired in the symmetry plane of the airfoil, which coincides with the symmetry line of the central bump for the SCB configurations. Differently, the schlieren data are affected by the integration of all the density gradient present along the line of sight. The similarity of the results that are nevertheless achieved, confirms that schlieren visualisations can be effectively used for studying the behaviour of transonic buffet on an airfoil, also in presence of 3D passive control systems.

The PIV data (see Fig. 12, 14, 15) show that also a diminished pulsation of the separated area is achieved when using SCBs (in addition to the reduced SW oscillation range). Although there is a small increase in the extent of the separated area in presence of the best behaving SCB configuration, there is an evident reduction of the extent of the shear layer area compared to the clean configuration.

To further investigate the difference in behavior among the various SCB configurations, PIV data on a spanwisechordwise plane of measurements have been used as well. This FOV proved to be particularly meaningful for the understanding of the mutual interaction of the different bumps and the effect of changing their separation distance. Figure 16 shows that the oblique shockwaves, originating at the leading edge of the 3D SCBs, curve around the bumps. These flow structures, together with the vortex production in the rear part of the bumps are considered responsible for the different results obtained when changing the spacing between the SCBs. The vortex production, although shown in the oil flow visualization in Fig. 1 (left), has not been investigated in detail in this study because of the distance of the plane of measurement above the separated area. To address this aspect, in future, experiments in the verticalchordwise plane of measurements at different spanwise positions should be carried out.

The spanwise-chordwise data have also allowed the investigation of the two dimensionality of the flow field. The results show a spanwise coherence of the quasi-normal moving shockwave for the $\Delta \mathrm{x}_{\mathrm{SCB}}=25 \%$ c configuration, notwithstanding the presence of the 3D control systems. Additional 3D SW structures arise for a wider spacing of the bumps.

In conclusion this study demonstrates the feasibility to control transonic buffet using 3D SCBs, and confirms the importance of the spacing parameter. In order to reduce their possible detrimental behaviour in off-design conditions (although very limited compared to 2D SCBs as described in [22]), an adaptive implementation (see Ref. [23]) of these devices on future vehicles can be suggested.

\section{Acknowledgments}

This work has been carried out as part of the project HOMER (Holistic Optical Metrology for Aero-Elastic Research), funded by the European Commission, program H2020 under Grant No. 769237.

The authors acknowledge Manfredi Messina for having helped with the realization of the SCBs and setting up part of the experiments.

\section{References}

[1] Hilton, W.F., and Fowler, R. G., "Photographs of Shock Wave Movement," NPL R\&M No. 2692, 1947.

[2] Lee, B. H. K., "Transonic buffet on a supercritical aerofoil," The Aeronautical Journal, 94(935) 143-152, 1990.

-[3] Jacquin, L., Molton P., Deck, S., Maury, B., and Soulevant, D., "Experimental study of shock oscillation over a transonic supercritical profile," AIAA Journal, 47(9):1985-1994, 2009.

- [4] Hartmann, A., Feldhusen, A., and Schröder, W. "On the interaction of shock waves and sound waves in transonic buffet flow," Physics of Fluids, 25(2):026101, 2013.

[5] Crouch, J.D., Garbaruk, A., Magidov, D., and Travin, A., "Origin of transonic buffet on aerofoils," Journal of Fluid Mechanics, 628:357-369, 2009.

[6] Giannelis, N. F., Vio, G.A., and Levinski, O., "A review of recent developments in the understanding of transonic shock buffet," Progress in Aerospace Sciences, 92:39 - 84, 2017.

[7] Caruana, D., Mignosi, A., Robitaillie, C., and Correge, M., "Active control of transonic buffet flow," Flow, Turbulence and Combustion, 824:312 - 351, 2003. 
- [8] Caruana, D., Mignosi, A., Correge, M., Le Pourhiet, A. and Rodde, A. M., "Buffet and buffeting control in transonic flow," Aerospace Science and Technology, 9(7):605 - 616, 2005.

[9] D’Aguanno, A., Schrijer, F. F. J., and van Oudheusden, B. W., "Transonic buffet control by means of upper gurney flaps," Proceedings of the 54th International Conference on Applied Aerodynamics (3AF 2019), March 25-27, 2019, Paris, France, 2019.

- [10] Bruce, P. J. K., and Colliss, S. P., "Review of research into shock control bumps," Shock Waves, 25(5):451-471, 2015.

-[11] Colliss, S. P., Babinsky, H., Nübler, K., and Lutz, T., "Vortical structures on three-dimensional shock control bumps," AIAA Journal, 2338-2350, 2016.

-[12] Ogawa, H., Babinsky, H., Pätzold, M., and Lutz, T., "Shock-wave/boundary-layer interaction control using three-dimensional bumps for transonic wings," AIAA Journal, 46(6), 1442-1452, 2008.

-[13] Holden, H., and Babinsky, H., "Shock/boundary layer interaction control using 3D devices," In 41st Aerospace Sciences Meeting and Exhibit, 2003.

- [14] Bruce, P. J. K., and Babinsky, H., "Experimental study into the flow physics of three-dimensional shock control bumps," Journal of Aircraft, 49(5), 1222-1233, 2012.

-[15] Mayer, R., Lutz, T., and Krämer, E., "Numerical Study on the Ability of Shock Control Bumps for Buffet Control," AIAA Journal, 1978-1987, 2018.

- [16] Eastwood, J. P., and Jarrett, J. P., "Toward designing with three-dimensional bumps for lift/drag improvement and buffet alleviation," AIAA Journal, 50 (12) 2882-2898, 2012.

[17] Bogdanski, S., Nübler, K., Lutz, T., and Kramer, E., "Numerical investigation of the influence of shock control bumps on the buffet characteristics of a transonic airfoil," New Results in Numerical and Experimental Fluid Mechanics IX, Springer, 2332, 2014.

-[18] König, B., Pätzold, M., Lutz, T., Krämer, E., Rosemann, H., Richter, K., and Uhlemann, H., "Numerical and Experimental Validation of Three Dimensional Shock Control Bumps," Journal of Aircraft, Vol. 46, No. 2, 675-682, 2009.

[19] D’Aguanno, A., Schrijer, F. F. J., and van Oudheusden B. W., "Study of upstream travelling waves in transonic Buffet," Proceedings of the 13th International Symposium on Particle Image Velocimetry (ISPIV 2019), July 22-24, 2019, Munich, Germany, 2019

[20] Schrijer, F. F. J., Solana Perez, R., and van Oudheusden B. W., "Investigation of transonic buffet using high speed PIV," Proceedings of the 5th International Conference on Experimental Fluid Mechanics, Munich, Germany, July 2-4, 2018.

[21] Humble R.A., "Unsteady Flow Organization of a ShockWave/Boundary Layer Interaction," Ph.D. thesis, Delft University of Technology, 2009.

[22] Mayer, R., Lutz, T., Krämer, E., and Dandois, J., "Control of Transonic Buffet by Shock Control Bumps on Wing-Body Configuration," Journal of Aircraft, 56(2), 556-568, 2019.

-[23] Gramola, M., Bruce, P. J. K., and Santer, M., "Experimental FSI study of adaptive shock control bumps," Journal of Fluids and Structures, 81, 361-377, 2018. 